\title{
3D modelling and measurements of historical violins
}

\author{
Piercarlo Dondi ${ }^{1}$, Luca Lombardi ${ }^{2}$, Marco Malagodi ${ }^{1,3}$, Maurizio Licchelli ${ }^{4}$ \\ ${ }^{1}$ University of Pavia, Arvedi Laboratory of Non-Invasive Diagnostics, via Bell'Aspa 3, 26100 Cremona, Italy \\ ${ }^{2}$ University of Pavia, Department of Electrical, Computer and Biomedical Engineering, via Ferrata 5, 27100 Pavia, Italy \\ ${ }^{3}$ University of Pavia, Department of Musicology and Cultural Heritage, Corso Garibaldi 178, 26100, Cremona, Italy \\ ${ }^{4}$ University of Pavia, Department of Chemistry, Via Taramelli 12, 27100, Pavia, Italy
}

\begin{abstract}
Measuring historical violins provides crucial information about the morphology of the instruments, useful both for researchers and violin makers. Generally, these measures are taken manually using a calliper, but they can be repeated only occasionally due to both the restricted access to these precious instruments and the need of avoiding accidental damages to the wood or to the varnishes. In this work, we describe and assess the accuracy of a protocol for the acquisition and creation of high quality 3D models of violins, suitable for taking accurate measurements. Six historical violins of $17^{\text {th }}-18^{\text {th }}$ centuries, kept in "Museo del Violino" in Cremona (Italy), were used as test set. The quality of the final outcomes was checked comparing measures taken on the 3D meshes with the correspondent ones taken by calliper on the original instruments. Finally, a comparison between the sound board of the instruments were performed.
\end{abstract}

Section: RESEARCH PAPER

Keywords: 3D scan; 3D measurement; historical violins

Citation: Piercarlo Dondi, Luca Lombardi, Marco Malagodi, Maurizio Licchelli, 3D modelling and measurements of historical violins, Acta IMEKO, vol. 6, no. 3, article 5, September 2017, identifier: IMEKO-ACTA-06 (2017)-03-05

Section Editor: Sabrina Grassini, Politecnico di Torino, Italy

Received March 8, 2017; In final form May 25, 2017; Published September 2017

Copyright: (C) 2017 IMEKO. This is an open-access article distributed under the terms of the Creative Commons Attribution 3.0 License, which permits unrestricted use, distribution, and reproduction in any medium, provided the original author and source are credited

Funding: This work was supported by Fondazione Cariplo [Research project “Opificio del Suono"], Regione Lombardia [POR Ob. 2 - FSE $2007 / 2013$ research project $n^{\circ} 61385772$ "VIVA MUSICA"] and Fondazione Arvedi Buschini of Cremona

Corresponding author: Piercarlo Dondi, e-mail: piercarlo.dondi@unipv.it

\section{INTRODUCTION}

Studying the morphology of a violin is a complex but important task. Many kinds of information can be retrieved and measured, such as the external dimensions, the internal thicknesses or even the impact of varnishes on the acoustic [1]. These data are particularly valuable in the case of historical violins, not only for researchers, but also for violin makers, that can use them as reference for the making of new instruments. Due to the importance of these data, accurate measures are always needed.

In this work, we focus on the measurement of the external surfaces of the violins, a task that, up to now, is only performed by the manual use of a calliper. Even if this procedure is standard with modern instruments and can be repeated several times, it can be performed only occasionally on historical ones. This is not only due to the restricted access but also to the need of limiting the direct contact of the calliper to its bare essential, in order to not ruin the ancient varnishes or even (in the worst scenario) chip the wood. The varnishes could represent a limit in the application of mechanical measurements because of their historical importance, brittleness and thickness [2]. Moreover, damages on the varnishes could be a key factor of the material degradations, especially biological ones, with a consequent requirement of a restoration work [3], [4]. A faithful 3D model of a violin can be very helpful in this field [5]. The 3D model allows to easily repeat measures several times without risks and also reach areas nearly inaccessible on the original instrument without opening it (e.g. the highness of the belly). However, the digitalization of a historical violin is a difficult process: the complex morphology and the presence of a great number of small details require a very high accuracy and degrees of freedom during the scanning phase [6]; moreover, the highly reflective varnishes complicates the use of some models of laser scanners or other scanning technologies, such as photogrammetry [7]. 
In one of our previous works, presented at MetroArcheo2016 [8], we discussed a protocol for the creation of accurate 3D models of historical violins using a laser scanner, and we tested it on the famous Antonio Stradivari "Cremonese" (1715). This article extends that work, the main improvements involve: I) the acquisition of other five important instruments of the $17^{\text {th }}-18^{\text {th }}$ centuries held in "Museo del Violino" of Cremona (Italy); II) a more complete assessment of the accuracy of the models; III) a set of comparisons between the shapes of the instruments.

The paper is organized as follows: Section 2 describes the acquisition and the modelling protocol; Section 3 discusses the measurement problem and the tests performed; Section 4 draws the conclusions.

\section{ACQUISITION AND 3D MODELING}

The digitalization of historical artworks is becoming a standard practice in many museums and archaeological sites [9]. Different imaging technologies are available (laser scanner, photogrammetry, stereo-cameras and so on). The final choice depends on the characteristics of the object to acquire [10]. These different techniques may be merged to improve the quality of the reconstructed models. As an example both photogrammetry and 3D scanning were used to create a model of the Donatello's "Maddalena" [11]. Mathys et al. presented a comparison among the different techniques, including also Computer Tomography; as a conclusion, the authors suggest using laser scanners when only external surfaces are needed and Computer Tomography (CT) if it is necessary to acquire internal structures [12]. Also, the possibility to use low cost devices, such as the Kinect, has been demonstrated for the reconstruction of excavation sites [13].

As previously stated, the acquisition of historical violins is particularly critical due to their complex morphology and the reflectivity of the varnishes. For these reasons, the digitalization of those instruments is generally obtained by a CT-scan. This is surely the most precise existing scanning technology; it can acquire both external and internal surfaces of an instrument and provides important information, such as the thickness of the ribs or the presence of internal damages and repairs [14]. Recently, the Smithsonian institution adopted the CT-scan for the study of seven Stradivari violins and proposed a detailed protocol for their acquisition and modelling. They also demonstrated the usability of such models for measurement tasks [15].

The use of laser scanners is instead very limited, since they can acquire solely the external surfaces. Only some notable cases are described in literature, such as The Strad 3D project [7] (however, that uses in combination also CT-scan data) or the analysis of elastic deformation performed on Guarneri del Gesù's "Cannone" (1734) [16]. Nevertheless, some advantages of this technology with respect to CT-scans must not be neglected: it is more affordable; it only needs a single operator to perform the acquisition instead of a team of experts; and it can be used directly in the museum without the need to move the instrument. Furthermore, in our field of interest the limitation to the external surface is not critical, since most of the important measures involve the outside of the violin.

Figure 1 provides an overview of our procedure. We shall now describe in details the various steps.

\subsection{Scanning}

Even if an optimal solution does not exist, some characteristics are crucial in the choice of the scanner to adopt:

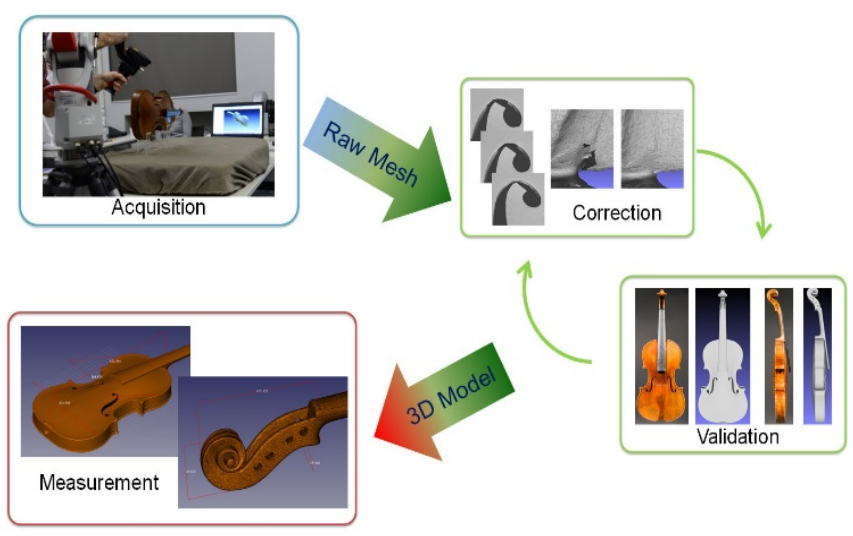

Figure 1. Overall schema of the proposed procedure of acquisition, 3D modelling and measurement of a violin.

I) a high degree of freedom, to reach all the surfaces of the instrument; II) a high accuracy, to acquire as many details as possible. For these reasons, we chose a "RS3 Integrated Scanner" (a linear laser scanner with a stated accuracy of 30 $\mu \mathrm{m})$ mounted on a mobile arm with 7 degrees of freedom (Romer Absolute Arm 7-Axis "SI") both produced by Hexagon Metrology. As scanning software, we used the PolyWorks Suite provided with the scanner.

Since wood instruments are sensible to abrupt variations in humidity and temperature that can alter their morphology [17], the working laboratory was always maintained at the same climatic condition of the showcases of the museum, a temperature of $20^{\circ}$ and a humidity of $50 \%$. Before the scanning, all the mobile parts of the violin that can interfere with the acquisition are removed: the tailpiece, the bridge and the chin rest because they hide part of the belly; the pegs because they partially hide the outer wall and the pegbox floor of the head; the strings because they can alter the light beam of the scanner. Their loss is acceptable, they are not strictly relevant for measurements and generally they are not the original ones (nearly all the mobile parts of historical violins have been substituted during the centuries).

Each instrument was acquired in four steps, generating many partial scans that will be aligned and connected during the elaboration phase: left side of the body, right side of the body, front side of the head, back side of the head. Two supports in Plexiglas, a vertical one (Figure 2a) and a horizontal one (Figure $2 \mathrm{~b}$ ), were designed to place the instrument in the optimal positions for reaching as much detail as possible. The vertical support was used for the body (better for reaching the internal edges of the f-hole and the part of the top plate under the fingerboard with the laser); the horizontal one for the head (better for reaching the pegbox floor, the peg holes and the throat of the scroll). Clamps covered with gum were used to maintain a stable position of the violins during the scanning

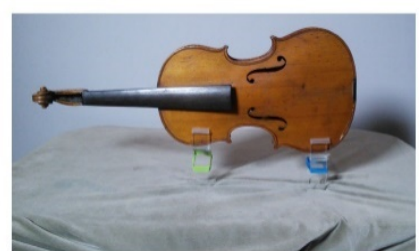

(a)



(b)
Figure 2. Support used for the scanning process: (a) vertical; (b) horizontal. 
process without damaging the varnishes.

The acquisition takes around six hours of work to be completed.

\subsection{Correction and validation}

After the acquisition of the raw data, the resulting cloud of points is cleaned removing redundant or badly acquired scans. In this step, particular attention must be paid to the head of the violin, because overlapping scans are very frequent due to its small dimension and to the presence of a lot of details that requires multiple runs. Then, the cleaned cloud of points is triangularized and converted in a raw $3 \mathrm{D}$ mesh.

The refinement of the raw 3D mesh was performed by two open source 3D modelling software packages, Meshlab and Blender. The number of corrections varies from instrument to instrument; it generally depends on the state of the surface. In particular, the presence of abrupt changes in colour or of very dark areas can interfere with the laser and produce errors or loss parts. Despite these considerations there are some areas of the instruments that are always problematic to reach with the scanner: the internal part of the head, the throat of the scroll, the internal edges of edge holes. High resolution images, previously acquired with a Nikon D4 camera equipped with a $50 \mathrm{~mm}$ objective [18], [19], were used as reference for the reconstruction of these areas. The procedure is iterative, each correction is validated using the images and the supervision of the curator of the museum, and repeated until the level of accuracy is considered acceptable. This refinement step may take one to two weeks, depending on the quality of the scans.

It is important to highlight that, even if the adopted laser scanner has a stated accuracy of $30 \mu \mathrm{m}$, it was designed for objects and surfaces more regular and less detailed than a violin. Therefore, the high number of scans needed for acquiring the entire surface can frequently produce an oversampling, even after the cleaning step. For obtaining more suitable models that can be used efficiently without high expensive hardware, we introduced a decimation step (quadric edge collapse decimation) during the correction phase. This simplification is focused only in those areas that present a clear oversampling, but of course, a decimation can alter the morphology of the object and then the accuracy of the measures. The following measurement tests were designed to prove that also these simplified 3D models are suitable for our measuring purposes.

\section{MEASUREMENTS}

Six historical violins were digitalized using the defined protocol: five made by Antonio Stradivari ("Clisbee" (1669), "Hellier" (1679), "Joachim-Ma (1714)", "Cremonese (1715)" and "Vesuvius" (1727)) and one made by Guarneri del Gesù ("Principe Doria" (1734)). This is an exhaustive test set, since each instrument has a peculiar history and was undergone to different restoration processes during the centuries that altered surfaces in different ways. Thus, even with a small test set, we could assess the quality of our acquisition method in heterogeneous conditions.

\subsection{Calliper vs 3D}

Taking geometrical measures on a violin is a complex task. The rounded shapes and the imperfect symmetry makes it difficult to obtain accurate and reproducible values. Moreover, the definition of the areas to be measured is not always univocal and can depend on subjective choices. To assess the accuracy of the acquired 3D models, we asked the curator of the museum to take a series of linear measures with a digital calliper (with a stated accuracy of $20 \mu \mathrm{m}$ ) on the original instruments. We subsequently replied the same measures (always with the supervision of the curator) on the corresponding 3D models using FreeCAD, an open source CAD software. Twenty-five metrics, typically used by violin makers and distributed on the entire surface of the instrument, were considered.

Table 1 shows the absolute and relative difference between calliper and 3D measures for each of the six violins. As can be seen, the quality of the 3D models, even if simplified, is high. In all the cases the overall average difference between calliper and $3 \mathrm{D}$ goes from $0.11 \mathrm{~mm}$ to $0.14 \mathrm{~mm}$. It is interesting to analyse separately the various "macro areas" of the instrument (the back plate, the table, the ribs, the f-holes and the scroll), to check if there are different levels of accuracy among them, due to inaccuracies in acquisition or to alterations generated by the elaboration of the meshes. The graphic in Figure 3 summarizes the average absolute difference for these zones and shows that no area is constantly more accurate than the others, with the only exception of the ribs. This behaviour proves that the accuracy of the model is quite uniform over the whole surface; the oscillations are compatible with random uncertainties due to the manual use of the calliper on a rounded surface. Instead, measures on the ribs are always very stable since they are easier to take.

The accuracy of the model is sufficient for most of the cases and for the needs of violin makers. However, if we want to perform high accuracy comparisons between instruments we need to check if an average difference of $0.14 \mathrm{~mm}$ is critical or not. For performing this verification, we computed for all the metrics the difference in terms of percentage (D) between each measurement taken with the calliper $\left(\mathrm{M}_{\mathrm{c}}\right)$ and the corresponding measurement taken from the $3 \mathrm{D}$ model $\left(\mathrm{M}_{3 \mathrm{D}}\right)$, as defined in (1).

$D=\frac{\left|M_{C}-M_{3 D}\right|}{M_{C}}$.

The graph reporting the mean percentage differences (Figure 4) shows clearer trends if compared to the graph reporting the mean absolute differences (Figure 3). The four parts that compose the body (back, table, f-holes and ribs) have always low average values that go from $0.05 \%$ to $0.28 \%$. On the contrary, measurements taken on the scroll show a greater variability (from $0.43 \%$ to $0.81 \%$ ). In particular, the scroll volute top width is very problematic, as can be seen in Table 1. As for the ribs, also for the scroll the areas to measure are small, however, the heights of the ribs are very easy to measure (the surface is perfectly straight in those areas), whereas the scroll is very rounded, and therefore it is more complex to find

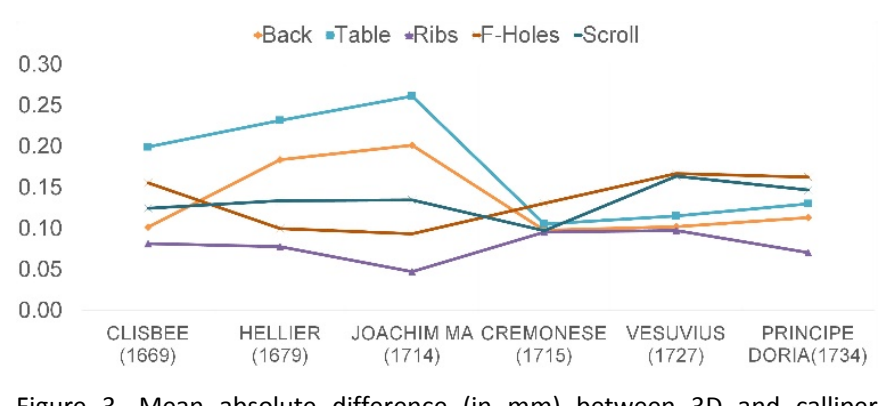

Figure 3. Mean absolute difference (in $\mathrm{mm}$ ) between 3D and calliper measures taken on the five main macro areas of the violins. 
Table 1. Absolute and percentage differences between measures taken by digital calliper on the violin and by CAD software on 3D model.

\begin{tabular}{|c|c|c|c|c|c|c|c|c|c|c|c|c|}
\hline \multirow[t]{2}{*}{ Metric } & \multicolumn{2}{|c|}{$\begin{array}{l}\text { Clisbee } \\
(1669)\end{array}$} & \multicolumn{2}{|c|}{$\begin{array}{l}\text { Hellier } \\
(1679)\end{array}$} & \multicolumn{2}{|c|}{$\begin{array}{c}\text { Joachim-Ma } \\
(1714)\end{array}$} & \multicolumn{2}{|c|}{$\begin{array}{c}\text { Cremonese } \\
(1715)\end{array}$} & \multicolumn{2}{|c|}{$\begin{array}{c}\text { Vesuvius } \\
\text { (1727) }\end{array}$} & \multicolumn{2}{|c|}{$\begin{array}{c}\text { Principe Doria } \\
\text { (1734) }\end{array}$} \\
\hline & $\mathrm{mm}$ & $\%$ & $\mathrm{~mm}$ & $\%$ & $\mathrm{~mm}$ & $\%$ & $\mathrm{~mm}$ & $\%$ & $\mathrm{~mm}$ & $\%$ & $\mathrm{~mm}$ & $\%$ \\
\hline Back length & 0.03 & 0.01 & 0.12 & 0.03 & 0.07 & 0.02 & 0.00 & 0.00 & 0.08 & 0.02 & 0.05 & 0.02 \\
\hline Back max higher width & 0.03 & 0.02 & 0.16 & 0.09 & 0.32 & 0.19 & 0.12 & 0.06 & 0.18 & 0.11 & 0.03 & 0.02 \\
\hline Back min width C-bouts & 0.10 & 0.10 & 0.20 & 0.17 & 0.07 & 0.06 & 0.19 & 0.18 & 0.11 & 0.10 & 0.22 & 0.21 \\
\hline Back max lower width & 0.25 & 0.13 & 0.26 & 0.12 & 0.35 & 0.17 & 0.08 & 0.05 & 0.04 & 0.02 & 0.15 & 0.07 \\
\hline Table length & 0.11 & 0.03 & 0.25 & 0.07 & 0.05 & 0.01 & 0.04 & 0.00 & 0.19 & 0.05 & 0.18 & 0.05 \\
\hline Table max higher length & 0.25 & 0.16 & 0.26 & 0.15 & 0.30 & 0.18 & 0.04 & 0.00 & 0.00 & 0.00 & 0.02 & 0.01 \\
\hline Table min width C-bouts & 0.26 & 0.26 & 0.28 & 0.25 & 0.23 & 0.22 & 0.29 & 0.27 & 0.08 & 0.07 & 0.19 & 0.17 \\
\hline Table max lower width & 0.18 & 0.09 & 0.14 & 0.07 & 0.46 & 0.23 & 0.05 & 0.05 & 0.19 & 0.09 & 0.14 & 0.07 \\
\hline Ribs height lower bouts & 0.05 & 0.16 & 0.20 & 0.62 & 0.09 & 0.28 & 0.03 & 0.00 & 0.05 & 0.17 & 0.00 & 0.00 \\
\hline Ribs height lower corners & 0.17 & 0.58 & 0.01 & 0.03 & 0.04 & 0.13 & 0.03 & 0.00 & 0.28 & 0.92 & 0.01 & 0.04 \\
\hline Ribs height upper corners & 0.01 & 0.04 & 0.10 & 0.30 & 0.05 & 0.15 & 0.17 & 0.63 & 0.05 & 0.16 & 0.14 & 0.49 \\
\hline Ribs height upper bouts & 0.10 & 0.35 & 0.01 & 0.02 & 0.01 & 0.04 & 0.15 & 0.33 & 0.01 & 0.03 & 0.13 & 0.46 \\
\hline Diapason & 0.14 & 0.07 & 0.02 & 0.01 & 0.03 & 0.02 & 0.07 & 0.05 & 0.09 & 0.05 & 0.29 & 0.15 \\
\hline F-holes upper lobes distance & 0.10 & 0.29 & 0.07 & 0.19 & 0.12 & 0.31 & 0.12 & 0.23 & 0.15 & 0.35 & 0.16 & 0.41 \\
\hline F-hole notches distance & 0.27 & 0.40 & 0.04 & 0.06 & 0.23 & 0.32 & 0.29 & 0.40 & 0.20 & 0.27 & 0.27 & 0.38 \\
\hline F-holes lower lobes distance & 0.17 & 0.17 & 0.20 & 0.19 & 0.15 & 0.14 & 0.20 & 0.18 & 0.11 & 0.10 & 0.02 & 0.02 \\
\hline F-hole right length & 0.19 & 0.26 & 0.23 & 0.31 & 0.02 & 0.02 & 0.01 & 0.00 & 0.37 & 0.50 & 0.18 & 0.25 \\
\hline F-hole left length & 0.07 & 0.09 & 0.04 & 0.05 & 0.01 & 0.01 & 0.09 & 0.13 & 0.07 & 0.09 & 0.06 & 0.08 \\
\hline Scroll length & 0.13 & 0.13 & 0.03 & 0.02 & 0.02 & 0.02 & 0.02 & 0.00 & 0.17 & 0.16 & 0.07 & 0.06 \\
\hline Scroll eyes width & 0.13 & 0.32 & 0.16 & 0.39 & 0.14 & 0.35 & 0.00 & 0.00 & 0.05 & 0.11 & 0.19 & 0.50 \\
\hline Scroll neck heel & 0.05 & 0.20 & 0.20 & 0.76 & 0.06 & 0.24 & 0.12 & 0.39 & 0.18 & 0.69 & 0.15 & 0.56 \\
\hline Scroll volute height & 0.05 & 0.10 & 0.16 & 0.32 & 0.17 & 0.35 & 0.26 & 0.61 & 0.44 & 0.87 & 0.00 & 0.01 \\
\hline Scroll first turn height & 0.24 & 0.64 & 0.12 & 0.30 & 0.02 & 0.04 & 0.14 & 0.26 & 0.09 & 0.22 & 0.09 & 0.23 \\
\hline Scroll volute top width & 0.22 & 2.11 & 0.03 & 0.29 & 0.27 & 2.45 & 0.07 & 0.85 & 0.22 & 2.13 & 0.44 & 3.96 \\
\hline Scroll max length over nut & 0.06 & 0.26 & 0.23 & 0.91 & 0.27 & 1.10 & 0.08 & 0.40 & 0.00 & 0.01 & 0.09 & 0.37 \\
\hline Average & 0.13 & 0.28 & 0.14 & 0.23 & 0.14 & 0.28 & 0.11 & 0.20 & 0.14 & 0.29 & 0.13 & 0.34 \\
\hline
\end{tabular}

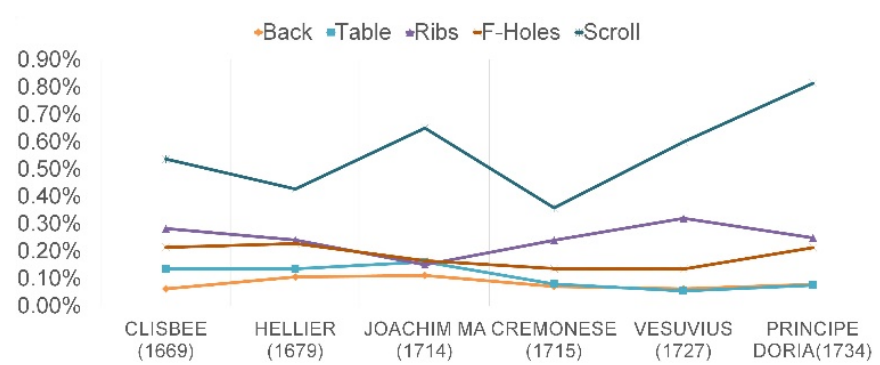

Figure 4. Mean percentage difference between 3D and calliper measures taken on the five main macro areas of the violins.

its correct reference points (both with calliper and with CAD software). Therefore, the uncertainty on the scroll is greater than on the ribs (see the two corresponding curves in Figure 3).

We can conclude that, even if measurements taken from the 3D models of the scroll are enough accurate for violin making purposes, it is better to not use them for making comparisons between violins; in this scenario it is more convenient to use only the body of the instruments.

\subsection{Comparison among violins}

The acquired Stradivarius violins are representative of the entire working period of the famous violin maker. Comparison of the bodies, and in particular of the soundboards, can give useful information about the evolution of Stradivari's construction techniques. In fact, Antonio Stradivari, during his long life, significantly modified his construction style, with variations in the volumes and sizes of the violins. For instance, one of the most important developments in Stradivari's work over the years was to flatten and straighten the arch, removing most of the recurve at the perimeter, and thereby providing a more responsive and powerful tone; with those modifications, the distances between the f-holes on the top changed, too [20].

Most of Stradivari's violins from the 'Golden Period' (after 1700s) show a fair symmetry between front and back, with generally less than a single millimetre discrepancy in height, and usually having the greater measurement on the front [21].

We extracted from the $3 \mathrm{D}$ models the external profile of the five soundboards and of the corresponding f-holes. The overlapping of these profiles allows to perform a fast and accurate comparison. Different measurements are taken again considering always the same metrics stated before.

Figure 5a shows the comparison between "Clisbee" and "Hellier". Even if only 10 years passed between the construction of the two instruments the differences are remarkable. "Clisbee" is clearly smaller than "Hellier": the length of the soundboard is $5.6 \mathrm{~mm}$ shorter, the max higher width (the maximum width of the upper half of the soundboard) is $13.9 \mathrm{~mm}$ narrower, the max lower width (the maximum width of the lower half of the soundboard) $16.5 \mathrm{~mm}$, and the min width at the C-bouts (the width of the narrowest part of the soundboard) $9.3 \mathrm{~mm}$. On the contrary, the dimensions of f-holes are not so different, only the lobes distance is lower in "Clisbee", due to its reduced width. This discrepancy can be explained considering that "Clisbee" is one of the first works of Antonio Stradivari, thus his style was still 

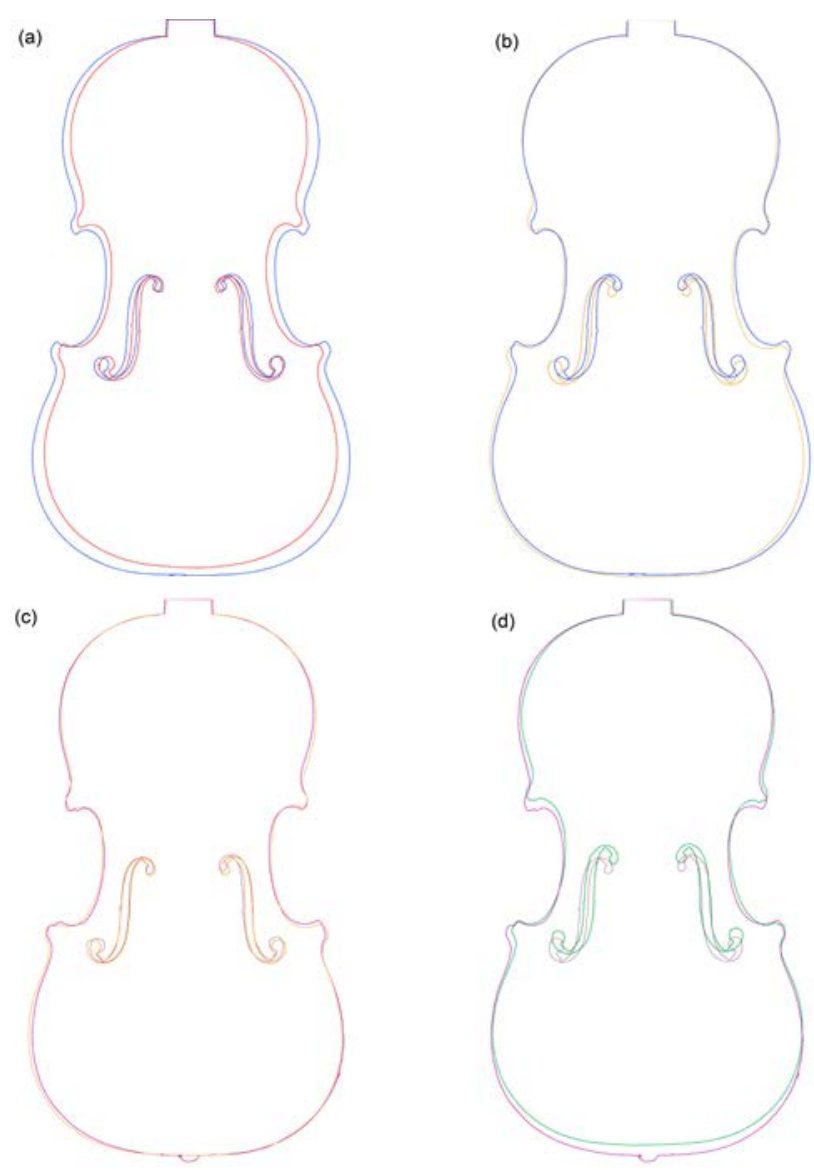

Figure 5. Comparison between violins: (a) "Clisbee" (red) vs "Hellier" (blue) (b) "Hellier" vs "Cremonese" (yellow); (c) "Cremonese" vs "Vesuvius" (violet); (d) "Vesuvius" vs "Principe Doria".

similar to that of his master, Nicola Amati.

In fact, the situation significantly changes comparing "Hellier" with "Cremonese". In this case, the shape of the two sound boards became quite similar, although the "Cremonese" was made 36 years later than the "Hellier" (Figure 5b). The total length is the same, the max higher width and the min width at the C-bouts are comparable, too. The only remarkable difference is in the max lower width, where "Hellier" is $3 \mathrm{~mm}$ wider. The dimensions of $\mathrm{f}$-holes are comparable, but there is a difference in the position, since in the "Cremonese" they are placed around $2.5 \mathrm{~mm}$ lower.

From this moment, the construction style remains quite stable. Dimensions of "Vesuvius", built 12 years later than "Cremonese", are very similar (Figure 5c). The differences are very limited, less than $1 \mathrm{~mm}$, with the only notable exception of the min width at the C-bouts: that in "Vesuvius" in $2.6 \mathrm{~mm}$ narrower that in "Cremonese".

Finally, as a final test, we compared the "Vesuvius", the last Stradivarius violin of our set, with "Principe Doria", a violin made only 7 year later by another violin maker, Guarneri del Gesù. This test is important since it can give information about difference in construction styles of makers who lived in the same historical period. The data retrieved are very interesting and the differences are remarkable (see Figure 4d). "Principe Doria" is $7.4 \mathrm{~mm}$ shorter than "Vesuvius", a length close to that of "Clisbee", built in the previous century. On the contrary, the other dimensions are comparable: there are 0.5 $\mathrm{mm}$ of difference between max lower widths of the two tables and $0.2 \mathrm{~mm}$ between the two min widths at the C-bouts. Only max lower width shows again a significant difference, since the "Principe Doria" is $1.5 \mathrm{~mm}$ narrower than "Vesuvius". Regarding the f-holes, the dimensions are quite the same, with few tenths of millimetres of difference. Their vertical position is different, but this is clearly due to the lower length of the "Principe Doria".

\section{CONCLUSIONS}

In this works we described a protocol for the acquisition of 3D models of historical violins, suitable for taking accurate measurements. The proposed approach was used to digitalize six historical violins kept in "Museo del Violino" in Cremona, five made by Antonio Stradivari and one by Guarneri del Gesù.

The accuracy of the obtained 3D models was assessed comparing measurements taken by a calliper on the original instruments and measures taken from CAD software on the 3D meshes. The overall accuracy was very high, showing an average discrepancy between calliper and 3D of around $0.14 \mathrm{~mm}$.

Comparisons performed among the six instruments proved to be useful to understand the evolution of the construction style of Stradivari and to underline differences between his work and that of Guarneri del Gesù.

Future developments involve the acquisition of instruments of other violin makers of the same historical period to perform further comparisons and a more in depth analysis of the $3 \mathrm{D}$ models that considers the morphology of their entire surface.

\section{ACKNOWLEDGEMENT}

We would like to thank "Fondazione Museo del Violino Antonio Stradivari", association "Friends of Stradivari" and "Distretto Culturale di Cremona" for collaboration. A special acknowledgment to the curator of the Museum Fausto Cacciatori.

\section{REFERENCES}

[1] F. Setragno, M. Zanoni, F. Antonacci, A. Sarti, M. Malagodi, T. Rovetta, C. Invernizzi, Feature-based analysis of the impact of ground coat and varnish on violin tone qualities, Acta Acustica 103 (1) (2017), pp. 80-93.

[2] C. Invernizzi, A. Daveri, T. Rovetta, M. Vagnini, M. Licchelli, F. Cacciatori, M. Malagodi, A multi-analytical non-invasive approach to violin materials: the case of Antonio Stradivari 'Hellier' (1679), Microchemical Journal 124 (2016) pp. 743-750.

[3] M. Clausi, G. M. Crisci, M. F. L. Russa, M. Malagodi, A. Palermo, S. A. Ruffolo, Protective action against fungal growth of two consolidating products applied to wood, Journal of Cultural Heritage 12 (1) (2011) pp. 28-33.

[4] M. Malagodi, C. Canevari, L. Bonizzoni, A. Galli, F. Maspero, M. Martini, A multi-technique chemical characterization of a Stradivari decorated violin top plate. Applied Physics A 112 (2013) pp. 225-234.

[5] E. Ravina, Parametrical 3d structural co-modelling of stringed instruments, Proc. of 20th International Congress on Acoustics, ICA 2010, Aug. 23-27, 2010, Sydney, Australia, p. 23-27.

[6] S. Zygmuntowicz, The strad 3d project: scientists, musicians, and violinmakers study three classic violins, The Journal of the Acoustical Society of America, 127 (3) (2010) pp 1791.

[7] L. Pinto, R. Roncella, G. Forlani, Photogrammetric survey of ancient musical instruments, Proc. of The International Archives of the Photogrammetry, Remote Sensing and Spatial Information Sciences, ISPRS Congress, 3-11 Jul. 2008, Beijing, China, Vol. 37, Part B5, pp. 308-313. 
[8] P. Dondi, L. Lombardi, M. Malagodi, M. Licchelli, Measuring Stradivari violin "Cremonese" (1715) by 3d modeling, Proc. of 2nd IMEKO International Conference of Metrology of Archaeology and Cultural Heritage, Oct. 19-21, 2016, Torino, Italy, pp. 29-33.

[9] F.Stanco, S. Battiato, G. Gallo, Digital imaging for cultural heritage preservation: analysis, restoration, and reconstruction of ancient artworks, CRC Press, 2011, ISBN 9781439821732.

[10] M. Hess, S. Robson, M. Serpico, G. Amati, I. Pridden, T. Nelson, Developing $3 \mathrm{~d}$ imaging programmes-workflow and quality control, J. Comput. Cult. Herit. 9 (1) (2015) pp. 1:1-1:11.

[11] G. Guidi, J. A. Beraldin, C. Atzeni, High-accuracy 3d modeling of cultural heritage: the digitizing of Donatello's "Maddalena", IEEE Transactions on Image Processing 13 (3) (2004) pp. 370_ 380.

[12] A. Mathys, J. Brecko, P. Semal, Comparing 3d digitizing technologies: what are the differences?, Proc. of Digital Heritage International Congress, Oct. 28-Nov. 1, 2013, Marseille, Vol. 1, pp. 201-204.

[13] M. Zollhöfer, C. Siegl, M. Vetter, B. Dreyer, M. Stamminger, S Aybek, F. Bauer, Low-cost real-time 3d reconstruction of large scale excavation sites, J. Comput. Cult. Herit. 9 (1) (2015), pp. 2:1-2:20.

[14] S. A. Sirr, J. R. Waddle, Use of ct in detection of internal damage and repair and determination of authenticity in high-quality bowed stringed instruments, RadioGraphics 19 (3) (1999) pp. 639-646.

[15] B. Frohlich, G. Sturm, J. Hinton, E. Frohlich, The secrets of the Stradivari string instruments. A non-destructive study of music instruments from the Smithsonian institution, the library of congress, and private collections. A pilot study of seven violins made by Antonio Stradivari in Cremona, Italy, between 1677 and 1709, The Materialise Group, Leuven, Belgium and Materialise, USA, Ann Arbor, MI, USA, Washington, DC (March 2009).

[16] M. Fioravanti, G. Goli, B. Carlson, Structural assessment and measurement of the elastic deformation of historical violins: The case study of the Guarneri "del Gesù" violin (1743) known as the 'Cannone', Journal of Cultural Heritage 13 (2) (2012) pp. 145153.

[17] G. Goli, M. Fioravanti, S. Busoni, B. Carlson, P. Mazzanti, Measurement and modelling of mass and dimensional variations of historic violins subjected to thermo-hygrometric variations: the case study of the Guarneri "del Gesù" violin (1743) known as the "Cannone", Journal of Cultural Heritage, 13 (3) Supplement (2012) pp. S154-S160

[18] P. Dondi, C. Invernizzi, M. Licchelli, L. Lombardi, M. Malagodi, T. Rovetta, Semi-automatic system for uv images analysis of historical musical instruments, Proc. of SPIE 9527, Optics for Arts, Architecture, and Archaeology V, June 21, 2015, Munich, Germany, pp. 95270H-1 - 95270H-9.

[19] P. Dondi, L. Lombardi, C. Invernizzi, T. Rovetta, M. Malagodi, and M. Licchelli. 2017. Automatic analysis of uv-induced fluorescence imagery of historical violins. Journal on Computing and Cultural Heritage 10 (2) (2017) pp. 12:1 - 12:13.

[20] H. K. Goodkind, Violin iconography of Antonio Stradivari, New York, Larchmont, 1972, ISBN 9780960049813.

[21] S. F. Sacconi, I 'segreti' di Stradivari, Libreria del Convegno, Cremona, 1972. 